\title{
Confined Brønsted Acid Catalysis
}

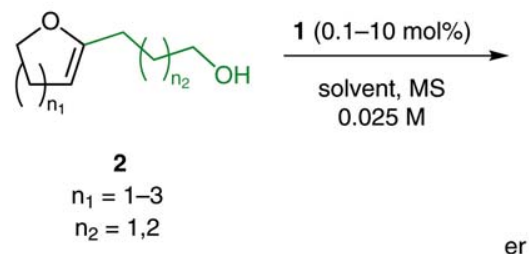

Selected examples:

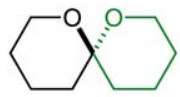

olean

$77 \%$ yield

er $=98: 2$

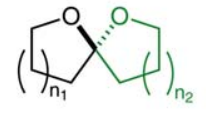

3

15 examples

$62-89 \%$ yield er from $95.5: 4.5$ to $98.5: 1.5$

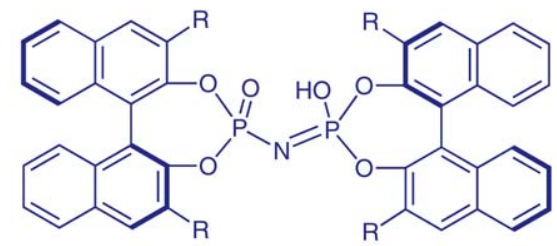

1

$\mathrm{R}=2,4,6-\mathrm{Et}_{3} \mathrm{C}_{6} \mathrm{H}_{2}$
Gategory

Organo- and

Biocatalysis

Key words

acetalization

confined Brønsted

acids

spiroacetals

SYNFACTinh
of the now previous acid catalysts

A

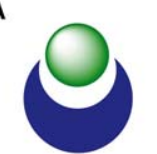

large substrate

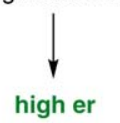

B

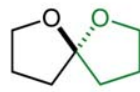

$62 \%$ yield er $=96: 4$

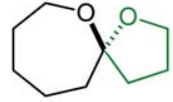

$88 \%$ yield er $=98.5: 1.5$

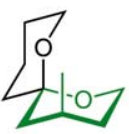

nonthermodynamic spiroacetal $\mathrm{dr}=7: 1$ $76 \%$ yield

(thermodynamic $\mathrm{dr}=1: 60$ )

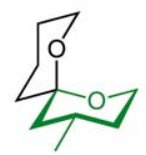

$\mathrm{dr}=100: 1$ $86 \%$ yield
Significance: Novel confined Brønsted acid catalysts based on a $C_{2}$-symmetric imidodiphosphoric acid motif were designed and synthesized. These catalysts possess an extremely sterically demanding chiral microenvironment. With catalyst $\mathbf{1}$, an asymmetric spiroacetalization of hydroxyenol ethers $\mathbf{2}$ to give small spiroacetals $\mathbf{3}$ has been developed. The spiroacetalization also provides a catalyst-controlled access to nonthermodynamic as well as thermodynamic spiroacetals. Importantly, to access imidodiphosphoric acids $\mathbf{1}$, only a single additional step is required compared to the corresponding phosphoric acids.
Comment: Despite numerous reports on Brønsted acid catalysis, particularly with phosphoric acids, reactions of small molecules still present a challenge. As shown schematically above, phosphoric acids typically give good results with relatively large substrates (A), while low enantioselectivity is obtained with small substrates due to insufficient interactions with the catalyst (B), and a resulting diversity of transition states that can be accommodated. In the case of confined acids, high enantioselectivity can be obtained with small substrates (C). In contrast, such catalysts may have difficulties in handling relatively large substrates, which may not fit into the catalytic cavity (D). 